\title{
AVALIAÇÃO DO RISCO DE QUEDAS EM IDOSOS CADASTRADOS EM UMA ESTRATÉGIA DE SAÚDE DA FAMÍLIA
}

Elaine Aparecida Lozano da Silva ${ }^{1}$, Larissa Borba André ${ }^{1}$, Beatriz Espanhol Garcia ${ }^{1}$, Estela Vidotto de Oliveira ${ }^{1}$, Ana Caroline Rippi Moreno ${ }^{1}$, Regina Celi Trindade Camargo ${ }^{2}$, Eliane Ferrari Chagas ${ }^{2}$, Ana Lúcia de Jesus Almeida ${ }^{2}$.

Universidade Estadual Paulista - UNESP, 'Pós-Graduação em Fisioterapia, ${ }^{2}$ Departamento de Fisioterapia, Presidente Prudente, SP. e-mail: elaine lozano@hotmail.com.

\section{RESUMO}

Os declínios causados pelo envelhecimento desenvolvem alterações no equilíbrio e capacidade funcional do idoso, predispondo ao surgimento de quedas. O objetivo do estudo é avaliar o risco de quedas em idosos. O estudo é de natureza transversal, com coleta por meio da aplicação do teste Timed up and go (TUGT) e a Escala de Equilíbrio de Berg. Estudo composto por 20 idosos com média de idade de 66,4 $\pm 9,7$ anos; na escala de Berg a pontuação obtida foi de 51,3 $\pm 2,8$ pontos, e no Teste TUGT, $9,90 \pm 3,14$ seg. Os participantes foram subdivididos em dois grupos de acordo com seu resultado no BERG: Grupo Maior e Grupo Menor. Na análise comparativa não houve diferença significativa, obtendo melhor resultado no TUGT o grupo maior $(p=0,1937)$. Diante do escore encontrado no presente estudo, caracteriza-se a população como sem risco de quedas. Desse modo destacando a importância de programas para prevenção e promoção da saúde na população idosa nas Estratégiad de Saúde da Família (ESFs) que atuam. Palavras-chave: Idoso, equilíbrio, envelhecimento, quedas, qualidade de vida.

\section{RISK ASSESSMENT OF FALLS IN ELDERLY REGISTERED IN THE FAMILY HEALTH STRATEGY}

\begin{abstract}
The declines caused by aging develop changes in balance and functional capacity of the elderly, predisposing to the emergence of drops. The objective of the study is to evaluate the risk of falls in the elderly. The study is cross-cutting, with collection through the test application Timed up and go (TUGT) and the Berg Balance Scale. This study was compound of 20 seniors with an average age of $66.4 \pm 9.7$ years; the Berg scale hit the score was $51.3 \pm 2.8$ points, and the TUGT test, $9.90 \pm 3.14$ seconds. In the comparative analysis there was no significant difference by getting shorter TUGT in the largest group $(p=0.1937)$. Given the scores found in this study characterized the population as without risk of falls. Thus highlighting the importance of programs for prevention and health promotion in the elderly in the Estratégia de Saúde da Família (ESF) through SUS.
\end{abstract}

Keywords: elderly, balance, aging, accidental falls, quality of Life.

\section{INTRODUÇÃO}

O frequente aumento da população de idosos é uma característica mundial, e Segundo o Instituto Brasileiro de Geografia e Estatística ${ }^{1}$, o Brasil vem se destacando entre os principais países com aumento da população idosa, sugerindo que no ano 2025, se classifique em sexto lugar entre os países mundiais com maior população de idosos.
O envelhecimento é um processo natural, progressivo e irreversível, em seu decorrer encontramos diversas modificações físicas e anatômicas que prejudicam a saúde do idoso e sua longevidade, tais como a mobilidade funcional e o equilíbrio corporal ${ }^{2}$. Tais modificações podem estar associadas a reduções de força, flexibilidade e massa muscular, bem como aos declínios dos órgãos sensoriais 
responsáveis pela manutenção do equilíbrio corporal $^{3}$.

O equilíbrio corporal é um processo complexo envolvendo diversos sistemas, como: integração da visão, sensação vestibular e periférica, comandos centrais, respostas neuromusculares, força muscular e do tempo de reação ${ }^{4}$. Observa-se um declínio significante no idoso em todas as partes desses sistemas, e estão ligados intrinsecamente ao desempenho funcional ${ }^{5}$.

O declínio no desempenho funcional pode afetar negativamente a qualidade de vida do idoso, necessitando de apoio e cuidados da família. No Brasil, fatores culturais, socioeconômicos e instabilidades no arranjo familiar, interferem diretamente no cuidado com o idoso, sendo comum auxílio de instituições de longa permanência para idosos. As intervenções realizadas no SUS possuem como principal objetivo a prevenção, promoção e reabilitação em saúde, com atenção especial sempre nas principais dificuldades dos idosos, a fim de contribuir para amenizar o impacto do envelhecimento, agravos em saúde e os riscos de quedas $^{6}$.

A ocorrência de quedas esta fortemente associada a taxas de mortalidade no Brasil, estando classificada em segundo lugar entre as principais causas de morte, cerca de $30 \%$ da população acima de 65 anos caem por ano, e essa porcentagem é diretamente proporcional ao avanço da idade ${ }^{7,8}$. Sendo assim as quedas representam um fator de grande impacto na saúde do idoso, apresentando como definição o "[...] deslocamento não intencional do corpo para um nível inferior a posição inicial, com incapacidades de correção em tempo hábil" ${ }^{\prime 9}$.

A busca pela avaliação do equilíbrio corporal é de suma importância para a elaboração de estratégias que visam proporcionar melhor qualidade de vida à população idosa. Dessa forma, o objetivo do estudo foi avaliar o risco de quedas em idosos cadastrados em uma Estratégia de Saúde da Família (ESF).

\section{METODOLOGIA}

Trata-se de um estudo transversal, aprovado pelo Comitê de Ética da Faculdade de Ciências e Tecnologia FCT/UNESP (CAAE 41034914.1.0000.5402), com idosos cadastrados em uma ESF do município de Presidente
Prudente. Os participantes leram e assinaram o Termo de Consentimento Livre e Esclarecido (TCLE).

Os critérios de inclusão foram: estar cadastrado na ESF São Pedro; aceitar as propostas dos residentes sobre a avaliação e assinar o TCLE. Os critérios de exclusão foram: apresentar algum tipo de intercorrência durante a aplicação dos testes, tais como indisposição, dores musculares, tonturas, vertigens e cefaleia; não concordar em assinar o TCLE; não compreender as orientações para realização do teste estipulado.

Foi realizado avaliação do equilíbrio corporal utilizando como instrumentos o teste Timed up and go (TUGT $)^{10}$ e a Escala de Equilíbrio de Berg (BERG) ${ }^{11-12}$. As avaliações foram realizadas pelos residentes em Fisioterapia da UNESP, campus de Presidente Prudente, na ESF, durante o horário dedicado a intervenção com esse grupo.

$O$ teste TUGT $^{10}$ tem como função a avaliação da mobilidade funcional e o equilíbrio corporal do indivíduo a partir do tempo gasto para realizar a tarefa de se levantar de uma cadeira sem auxílio dos braços, percorrer uma distância de 3 metros, contornar um cone posicionado para delimitar a metragem, retornar e sentar. $O$ desempenho na realização do teste está relacionado com o risco de quedas que ele pode possuir, sendo que um tempo superior a 13,5 segundos caracteriza maior risco de quedas. Este teste foi elaborado e adaptado a partir do Get-up and go test ${ }^{13}$.

O BERG ${ }^{10-11}$ tem como função avaliar o equilíbrio estático e dinâmico por meio de atividades funcionais. A escala é formada por 14 tarefas simples que fazem parte do cotidiano. Cada item tem cinco alternativas com escore de 0-4, sendo 56 a pontuação máxima, quanto maior a pontuação, melhor a relação com o equilíbrio. Para interpretação do teste seguimos o que foi descrito por Berg ${ }^{14}$, que utilizou como amostra indivíduos idosos que necessitavam fazer uso de dispositivo para marcha e haviam sofrido AVE, tendo como referência a seguinte pontuação: $\leq$ 45 pontos $=$ risco para quedas; $>45$ pontos $=$ sem risco para quedas ${ }^{15}$.

Os testes de equilíbrio TUGT e BERG são ferramentas confiáveis e de fácil aplicação, ambas podem auxiliar em intervenções que objetivam prevenção de quedas, principalmente na população idosa que apresentam maior suscetibilidade. 
A análise estatística foi realizada pelo programa PRISMA e expressos por valores de média e desvio padrão. Para correlação entre o desempenho dos testes, os participantes foram subdivididos em dois grupos de acordo com seu resultado no BERG, sendo os 10 maiores resultados formando o Grupo Maior, e os 10 menores o Grupo Menor. Foi utilizada a análise estatística Test $t$ não paramétrico. As diferenças entre os grupos foram consideradas significantes quando $p<0,05$.

\section{RESULTADOS}

O estudo foi composto por 20 idosos com média de idade de $66,4 \pm 9,7$ anos. Para o BERG obteve-se como resultado a média de $51,3 \pm 2,8$ pontos, sendo o maior valor encontrado de 56 pontos e menor valor de 45 pontos.

Em relação ao teste TUGT, a média de resultado foi de 9,90 $\pm 3,14 \mathrm{seg}$, sendo encontrado o maior valor de 21,9 seg e menor de 6,9 seg. Vale ressaltar que quanto menor o resultado melhor o desempenho dos participantes.

Foi realizada uma subdivisão entres os participantes, com objetivo de verificar a presença de resultados inferiores no Berg, e também no TUGT. Desse modo um grupo ficou com 10 participantes com os maiores valores de BERG, e o outro com 10 participantes com menores valores. Após foi realizada a análise de comparação entre os resultados do TUGT, pelo Teste t não paramétrico (Figura 1).

Não foi encontrada diferença significante entre os testes, porém foi encontrada diferença $(p>0,05)$ de aproximadamente $15 \%$ no grupo Menor, visto que quanto maior o resultado do TUGT, pior o desempenho.

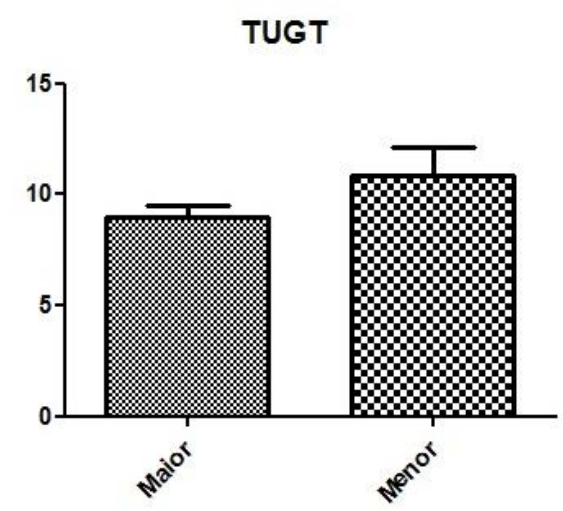

Figura 1. análise comparativa entre os grupos de maiores e menores valores no BERG.

\section{DISCUSSÃO}

A média dos valores encontrados no presente estudo foi $9,90 \pm 3,14$ seg para o teste TUGT e 51,3 $\pm 2,8$ pontos para a escala de equilíbrio de Berg. Desse modo de acordo com a classificação $25 \%$ da população apresentaram valores acima do escore do TUGT e $15 \%$ acima do escore de Berg ${ }^{12,13}$.

No estudo composto por com 36 idosas ativas, obtiveram como resultado $10,0 \pm 0,4 \mathrm{seg}$ para o TUGT e $51,3 \pm 0,6$ para o $\mathrm{BERG}^{16}$. Diante desse resultado, pode-se sugerir que a média alcançada dos participantes do presente estudo está similar a estudos com $100 \%$ da população ativa.

Os elevados valores encontrados podem ir de encontro justamente ao fato de que os participantes do estudo praticam atividade física regular realizada na ESF por fisioterapeutas residentes. A prática de atividade física oferece efeitos benéficos para a saúde do idoso, e em especial para a redução de quedas e dos declínios funcionais que prejudicam a marcha e o equilíbrio corporal ${ }^{17}$.

Com base na literatura ao correlacionar o BERG e o TUGT individualmente, verifica-se que idosos com maiores pontuações no Teste BERG realizam o TUGT em menor tempo ${ }^{12-14,18}$. Resultado semelhante ao encontrado no presente estudo, visto que os 10 participantes com maior resultado no Berg, obtendo média de $53,1 \pm 1,5$ concluíram o TUG em média de $8,7 \pm 1,5$ seg. Já o grupo com menor resultado no Berg, atingiram uma média de 49,6 $\pm 2,7$ e no TUGT $10,8 \pm 4,0$ seg.

O crescimento da população da terceira idade vem ocorrendo de forma acelerada e, portanto, diagnosticar e tratar os parâmetros clínicos associados com a redução da funcionalidade e quedas em idosos torna-se um grande desafio para a saúde pública, sendo assim de suma importância as intervenções fisioterapêuticas preventivas desenvolvidas nas ESF. Além disso, a ferramenta de avaliação Berg e TUGT possuem uma boa objetividade de testereteste e uma boa consistência interna, conseguindo diferenciar os idosos mais propensos a quedas, além de estar correlacionado a outros requisitos como possíveis déficits de equilíbrio e de mobilidade.

Diante dos dados expostos, pode-se concluir que os 20 participantes desse estudo, cadastrados na ESF São Pedro, apresentam baixos riscos de quedas e boa funcionalidade, 
destacando a importância de programas para prevenção e promoção da saúde na população idosa, como os oferecidos nesta ESF pelos residententes em Fisioterapia, pois esta intervenção pode estar diretamente relacionada com o bom desempenho nos testes. Outros estudos com populações com essas características, mas sedentários, pode vir a ratificar os resultados aqui apresentados.

\section{CONFLITO DE INTERESSES}

Os autores declaram não haver qualquer potencial conflito de interesses que possa interferir na imparcialidade deste trabalho científico.

\section{REFERÊNCIAS}

1. Instituto Brasileiro de Geografia e Estatística. Indicadores sociodemográficos e de saúde no Brasil. Rio de Janeiro, 2009. Disponível em: Acesso em: 5 ago. 2016.

2. Fagherazzi SB. Análise da influência de diferentes fatores sobre as pressões ventilatórias máximas em idosos no município de Porto Alegre [Dissertação]. Mestrado em Gerontologia Biomédica no Instituto de Geriatria e Gerontologia, Pontifícia Universidade Católica do Rio Grande do Sul, Porto Alegre, 2010.

3. Souza L, Coelho B, Freire B, Delevatti R, Rocanda C, Tiggemann C, Dias C. Comparação dos níveis de força e equilíbrio entre idosos praticantes de musculação e de hidroginástica. Rev Bras Ativ Fis e Saúde. 2014;19(5):647-8. DOI: http://dx.doi.org/10.12820/rbafs.v.19n5p647

4. Overstall PW. The use of balance training in elderly people with falls. Rev Clin Gerontol. 2003;13:153-61. DOI: http://dx.doi.org/10.1017/S0959259803013273

5. Rodrigues GD, Barbeito AB, Junior EDA. Prevenção de quedas no idoso: revisão da literatura brasileira. Rev Bras Prescr Fisiol Exerc. 2016;10(59):431-437.

6. Silvestre JA, Costa Neto MM. Abordagem do idoso em programas de saúde da família. Cad Saúde Públ. 2003;19(3):839-47.

DOI:

http://dx.doi.org/10.1590/S0102-

311X2003000300016

7. Bortoli CG, Pioveza MR, Piovesa EJ, Zonta MB. Equilíbrio quedas e funcionalidade em idosos com alteração da função cognitiva. Rev Bras Geriatr Gerontol. 2015;18(3). DOI: http://dx.doi.org/10.1590/1809-9823.2015.14057

8. Ansai JH, Glisoi SFN, Oliveira T, Soares AT, Cabral KN, Sera CTN. Revisão de dois instrumentos clínicos de avaliação para predizer risco de quedas em idosos. Rev Bras Geriatr Gerontol. 2014;17(1):177-89.
http://dx.doi.org/10.1590/S1809-

98232014000100017

9. Teixeira CS, Schmidt FC, Muraro MFR, Meereis ECW. Revalência do risco de quedas em idosos de uma instituição de longa permanência de Santa Maria (RS). Kirós Gerontol. 2014;17(1):45-56.

10. Gonçalves DFF, Ricci NA, Coimba AMV. Equilíbrio funcional de idosos da comunidade: comparação em relação ao histórico de quedas. Rev Bras Fisioter. 2009;13(4):316-23.

DOI:

http://dx.doi.org/10.1590/S1413-

35552009005000044

11. Gunter KB, White KN, Hayes WC, Snow CM. Functional mobility discriminates nonfallers from onetime and frequent fallers. J Gerontol Med Sci. 2000;55(11):672-6. DOI: http://dx.doi.org/10.1093/gerona/55.11.M672

12. Mathias S, Nayak USL, Isaacs B. Balance in the elderly patient: the "Get-up and Go" test. Arch Phys Med Rehabil. 1986;67:387-9.

13. Miyamoto ST, Lombardi Júnior I, Berg KO, Ramos LR, Natour J. Brazilian version of the Berg Balance Scale. Braz J Med Biol Res. 2004;37(9):1411-21. DOI: http://dx.doi.org/10.1590/S0100-

\section{X2004000900017}

14. Berg KO, Wood-Dauphinee SL, Williams Jl. Measuring balance in the elderly: validation of an instrument. Can J Publ Health. 1992;83:S7-S11.

15. Podsiadlo D, Richardson S. The Timed Up \& Go: A test of basic functional mobility for frail elderly persons. J Am Geriatr Soc. 1991;39:142-8. DOI: https://doi.org/10.1111/j.1532-5415.1991.tb01616.x

16. Neto JP, Raso W, Brito CAF. Mobilidade funcional em função da força muscular em mulheres idosas fisicamente ativas. Rev Bras Med Esporte. 2015;21(5):369-71. DOI:

http://dx.doi.org/10.1590/1517869220152105112756

17. Rodrigues JE, Gameleira AB, Barros JFP, Filho AVD, Gomes CAFP. Relação entre atividade física e equilíbrio em idosas de um grupo da terceira idade. Neurobiologia. 2012;75(1-2):141-146.

18. Vaz DP, Gazzola JM, Lança SM, Dorigueto RS, Kasse CA. Clinical and functional aspects of body balance in elderly subjects with benign paroxysmal positional vertigo. Braz J Otorhinolaryngol. 2013;79(2):150-7. DOI: http://dx.doi.org/10.5935/1808-8694.20130027 Recebido para publicação em 19/08/2016 Revisado em 25/08/2016

Aceito em 05/09/2016 